\title{
Erratum
}

\section{Erratum zum Beitrag: Rekonstruktion der aktiven Ellbogenbeugung durch bipolare Transposition des Musculus latissimus dorsi. Oper Orthop Traumatol 2009;21:115-25 (DOI 10.1007/s00064-009-1702-y)}

Andreas Gohritz, Marcus Spies, Jan Fridén, Karsten Knobloch, Andreas Steiert, Mehmet Altintas, Peter M. Vogt

Versehentlich wurde der Name des Autors Mehmet Ali Altintas unvollständig angegeben. Die korrekte Auflistung der Autorennamen lautet wie folgt:

Andreas Gohritz, Marcus Spies, Jan Fridén, Karsten Knobloch, Andreas Steiert, Mehmet A. Altintas, Peter M. Vogt. 